\title{
25 Research Soure \\ The gut microbiome is more stable in males than in females during the development of colorectal cancer
}

Haijun Liao

Sichuan University

Chaonan Li

Chengdu Institute of Biology

Yingwei Ai

Sichuan University

Yongping Kou ( $\nabla$ kouyp@cib.ac.cn )

Chengdu Institute of Biology https://orcid.org/0000-0002-9069-3255

\section{Research}

Keywords: colorectal cancer, gut microbiome, community assembly, co-occurrence network, sexdependent

Posted Date: January 22nd, 2020

DOI: https://doi.org/10.21203/rs.2.21627/v1

License: (9) (1) This work is licensed under a Creative Commons Attribution 4.0 International License. Read Full License 


\section{Abstract}

Background Gut microbial shifts have great potential to predict the risk of colorectal cancer, but how gut microbes respond to the development of colorectal cancer in males and females at the community scale is unknown. To address this question, we reanalyzed the dataset from a published project and grouped these data depending on sex into three groups, healthy, colorectal adenoma and carcinoma individuals, and community assembly and network patterns of gut microbes were evaluated by null model and cooccurrence network-based methods. Results The study showed that distinct changes in microbial a diversities and community composition were observed between the male and female gut. Specifically, the microbial a -diversities did not significantly change in the male gut $(P>0.05)$ but were lowered in the female gut $(P<0.05)$, suggesting that permanent species loss might occur in the female gut during the development of colorectal cancer. These distinct changes in microbial a -diversities and community composition between the male and female gut might result from host immunity mechanisms and different microbial community assemblies in males and females. Indeed, we found that the microbial community tended to be primarily stochastic in males, whereas it became increasingly deterministic in females with the development of colorectal cancer. Moreover, microbial co-occurrence associations tended to be more complicated when communities were primarily driven by stochastic processes in males; rare species were enriched in the co-occurrence network of the male gut, whereas key species loss was observed in the co-occurrence network of the female gut. Conclusions Our findings indicate that microbial communities in the male gut are more stable than those in the female gut and that microbial community assembly in the gut is sex-dependent with the development of colorectal cancer. Our study suggests that sexual dimorphism needs to be taken into account to better predict the risk of colorectal cancer based on microbial shifts.

\section{Background}

Colorectal cancer is highly associated with inflammatory bowel disease [1], and the dysbiosis of intestinal microbiota has been recognized as one of the drivers of inflammatory bowel disease [2] that alters the interactions between microbes and the mucosal immune system [3,4]. Therefore, intestinal microbes have been successfully developed as a clinical approach for colorectal cancer risk detection in recent years [5-9]. Gut microbes dynamically interact with intestinal epithelial cells [10,11]; therefore, microbial communities are likely altered with the development of colorectal cancer. In addition, microbial communities may also have adaptive strategies to resist lesion shocks and maintain the completeness of communities and functions. Previous studies reported that intestinal microbial aggregates are sexdependent [12-14], but the response mechanism during colorectal cancer development is still unclear.

Gut microbial communities can remain homeostatic in normal individuals [15]; however, this homeostasis will be broken by the development of colorectal cancer, which weakens the plastic adjustment of normal communities. Previous studies have demonstrated that the microbial communities in colorectal cancer patients deviate from those in normal individuals [16-18], and some studies have revealed how gut microbes change in healthy individuals compared to colorectal cancer patients at the taxonomic scale. 
However, the conclusions of these studies have not been consistent; for instance, microbes belonging to the Firmicutes phylum were detected with lower abundance in colorectal cancer patients in a previous study [19], whereas Firmicutes dominated in colorectal cancer patients in a recent study [20]. Moreover, the genera Bacteroides [21] and Fusobacterium [9, 22] were enriched, whereas Ruminococcus [21] and Bacteroides [20] were depleted in patients with colorectal adenoma or cancer. Unlike gastric cancer, which is probably solely derived from a single pathogenic bacterium, such as Helicobacterspp., colorectal cancer is involved in multiple microbial changes [16-18]. The gut microbiota is influenced by the age [23], diet style [24, 25] and genetic background of the host [26]; therefore, it is difficult to capture the full picture of microbial changes if we only focus on which taxon increased or decreased. Ecological insights concern microbial changes at the community scale, which may help us move beyond the current situation and reveal ecological response strategies of microbial communities to colorectal cancer.

Sexual divergences must be taken into account when detecting microbial changes during the development of colorectal cancer because sexual maturation, hormones, and the differences in the immune system and function profoundly affect microbe colonization in the gut [12-14]. Differences in initial colonization represent historic factors that likely contribute to how the microbial community responds to colorectal cancer, while sexual dimorphism may serve as a crucial long-term selection pressure that determines how microbial species aggregate [27]. Recent studies revealed that the gut microbial communities in males are distinguished from those in females in the face of external environmental pressures [28, 29], suggesting that males and females have distinct microbial aggregation strategies in the gut. Studies focusing on abdominal obesity-related disease [30], type I diabetes [31, 32], and major depressive disorder [33] have also demonstrated that sex-specific differences are important for shaping the gut microbial community with the development of related diseases. These results imply that sexual divergence is a primary factor in shaping the gut microbial community. Gut microbial changes and disease are highly associated with sex differences [12, 13]; however, the mechanism of species assembly with the development of colorectal cancer in males and females, with respect to ecological insights, is unknown.

Community assemblies describe microbial responses to environmental changes at the community scale and have been successfully applied in the microbial ecology of natural ecosystems [34]. Microbial community assembly is associated with both deterministic and stochastic processes [35]. Deterministic processes are involved in the ecological selection of both biotic and abiotic factors, which influence microbial assembly by changing the fitness between organisms and the environment and eventually altering community composition and species abundance [36]. Stochastic processes include dispersal, random birth, death and ecological drifts, which result in communities that are close to those produced by chance [37]. The balance between these two ecological processes could reflect how intestinal microbes respond to the development of colorectal cancer at the community scale; however, these processes have not been used to explain the variation in microbial communities with the development of colorectal cancer in males and females. A recent study indicated that colorectal cancer might influence microbial assembly indirectly by altering niche differentiation [38]. In soil microbial ecological research, lineages of bacteria can easily branch from a more extreme environment to colonize a more temperate environment, 
causing adapted lineages to colonize or accumulate in temperate environments, further resulting in greater diversity and higher stochastic assembly [34]. The gut microbiota is essential to complex ecological communities that have little fundamental difference from other ecological communities in nature, such as soils and lakes. However, whether ecological forces that shape gut microbial communities and the development of colorectal cancer are discrepant between males and females is unclear.

Gut microbes in communities mutually interact to form stable networks to serve specific ecological functions $[39,40]$. The enrichment or depletion of specific lineages in local communities might break the balance of the entire microbial ecosystem if these species are essential components in the ecological function network. Co-occurrence patterns are ubiquitous and particularly important in understanding microbial community structure, offering new insights into potential interaction networks among microbes at the system-level scale and revealing niche spaces shared by lineages in local communities [41-43]. Taxonomic changes that are induced by the development of colorectal cancer likely alter the topological features of the co-occurrence network and further change microbial ecological function profiles. By identifying the lineages with high topological importance in the network using topology-based analysis of large networks, it is likely that novel indicators that cannot be detected with traditional methods can be found. Comparing the topological properties of nodes (a node represents a species in network analysis) and networks can provide insights into the response mechanism of gut microbial communities to cancerinducing environmental changes.

In this paper, we compared the response mechanism of the gut microbial community at the community scale with the development of colorectal cancer in males and females using high-throughput sequencing data from the study of Zackular et al. (2014). Specifically, we downloaded 90 high quality fecal samples from public databases to represent the time series of colorectal cancer (healthy, colorectal adenoma and carcinoma). Two basic questions have been addressed: (i) Are the responses of the gut microbial community to colorectal cancer development gender-dependent? (ii) How is the microbial response to colorectal cancer development at the community scale determined based on ecological insights in males and females?

\section{Results}

\section{Landscape of microbial community}

Microbial $a$-diversities were detected without significant changes across the development of colorectal cancer in the male gut $(P>0.05)$, while colorectal cancer apparently lowered gut microbial diversity in females $(P<0.05)$ (Fig. 1C). Similarly, microbial composition also had no significant difference with colorectal cancer development proceeding in males, except for a slight divergence between healthy and cancer individuals $\left(R^{2}=0.063, P<0.05\right)($ Fig. $1 \mathrm{~A})$. However, significant community deviations were detected with the development of colorectal cancer in female individuals (Fig. 1B). Shared OTU counts among developmental stages of colorectal cancer in male individuals were higher than those in female 
individuals (Fig. 1D; Fig. 1E). Specific OTU counts increased with the development of colorectal cancer in male individuals (Fig. 1D) but decreased in female individuals (Fig. 1E). Shared functional gene counts were similar in male and female individuals (Fig. 1F; Fig. 1G). Although functional gene counts decreased with the development of colorectal cancer in both male and female individuals, changes in the female gut were greater than those in the male gut (Fig. 1F; Fig. 1G). In addition, species loss occurred in both males and females with the development of colorectal cancer (Additional file 1: Fig. S1; Fig. S2).

\section{Microbial community assembly}

Microbial community dispersion increased in male individuals (Fig. 2A) but decreased in female individuals (Fig. 2B) with the development of colorectal cancer. The observed microbial $\beta$-diversities in the male gut were significantly distinguished from the null distribution in healthy and adenoma individuals $(P$ $<0.001$ ), but no significant difference was detected in colorectal cancer patients ( $P=0.088$ ) (Fig. 2C). However, observed microbial $\beta$-diversities in the female gut were detected without significant deviations from the null distribution $(P>0.05)$ (Fig. $2 C$ ). The observed $\beta$-diversities increased with the development of colorectal cancer in male individuals but gradually decreased in female individuals (Fig. 2D). Values of standard effect size (SES) among development stages of colorectal cancer were detected without apparent changes compared to observed $\beta$-diversity in males (Fig. 2D). In contrast, the SES in female individuals dramatically decreased with the development of colorectal cancer (Fig. 2D).

\section{Microbial co-occurrence pattern}

The microbial meta co-occurrence network in the male gut included 5689 nodes and 90760 edges, while the meta co-occurrence network in the female gut contained 77167 associations among 5522 nodes (Fig. 3; Additional file 1: Tab. S1). Nodes in the male network were highly aggregated, but they tended to cluster into network modules in the female network (Fig. 3; Additional file 1: Tab. S1). Connections among network modules in the male network were stronger than those in the female network (Fig. 3). Firmicutes, Bacteroidetes, Proteobacteria and Actinobacteria were detected with high abundance $(>1 \%)$ in both networks, but Tenericutes (> 1\%) was only observed in the female network (Fig. 3).

With the development of colorectal cancer, links among network modules increased in the male network but decreased in the female network (Fig. 4). Node degree and betweenness centralities increased in the male network but decreased in the female network with the development of colorectal cancer (Fig. 5). Centrality-based tiers indicated a distinct response of core network nodes to colorectal cancer between males and females (Fig. 6). Microbes belonging to the Parcubacteria phylum were detected in the intermediate tier of the male adenoma network. Although Parcubacteria disappeared, Spirochaetes, Lentisphaerae and Planctomycetes arose in the male cancer network. The Synergistetes phylum was primarily located at the center of the female healthy network, but some of the members of this phylum 
migrated to the intermediate tier with colorectal cancer. Lentisphaerae and Acidobacteria were detected in the intermediate tier of the healthy female network, but they were excluded from the adenoma and cancer network (Fig. 6).

Principal component analysis of network topological properties revealed that network structures have little correlation with colorectal carcinoma in male individuals (Additional file 1: Fig. S3A). However, colorectal carcinoma deviated significantly from network structures in female individuals (Additional file 1: Fig. S3B). Topological properties including node count, edge count, average path length, cluster number, modularity and diameter significantly decreased, whereas network density increased with the colorectal cancer individuals in the female networks (Additional file 1: Fig. S4). In contrast, all topological properties were detected without significant changes in male networks across colorectal cancer (Additional file 1: Fig. S4).

\section{Discussion}

The human gut microbiome has been recognized to correlate with the development of colorectal cancer and has great potential to predict the risk and clinical status of colorectal cancer [6-9]. Previous studies have indicated that the human gut microbiome is sex-dependent [28]. However, it was unclear whether the ecological responses of gut microbial communities to colorectal cancer development were sexdependent. In this paper, to answer this question, we reanalyzed 90 fecal samples from the dataset of Zackular et al. (2014). The results revealed that the responses of gut microbial communities to colorectal cancer are sex-dependent, and the enrichment of rare species may contribute to the stability of microbial communities in the male gut, whereas species loss may be responsible for the vulnerable microbial communities in the female gut with the development of colorectal cancer. Therefore, sex needs to be considered for accurate prediction of the risk of colorectal cancer.

Diversity reflects the stability of the ecosystem, and previous studies have demonstrated that colorectal cancer could decrease gut microbial diversity [44]. This is consistent with the present study that species depleted in female individuals (Additional file 1: Fig. S2) may induce a-diversities that drastically decrease with the development of colorectal cancer. However, although species losses were also observed in males (Additional file 1: Fig. S1), the a- diversities were not significantly different during the development of colorectal cancer in males $(P>0.05)$. These results showed that the relationships between colorectal cancer and gut microbial diversity were sex-dependent. Moreover, species diversity and co-occurrence networks indicated that microbial communities were more vulnerable to colorectal cancer in female individuals than in male individuals with the development of colorectal cancer. Our results are consistent with the finding of Huang [45] on the sex-dependent changes in gut microbial communities during the development of hepatocellular carcinoma in mice, which demonstrated that the microbial community in females was more easily disordered than that in males. To explain the divergences between male and female individuals, we hypothesized that 'role effects' and 'compensatory effects' determine how the microbial community responds to colorectal cancer. The species lost with the development of colorectal cancer might exhibit low abundance, competition and growth rates [46] or 
contribute less to microbial functions in males, whereas depleted species are likely abundant and govern most ecological functions in females. The divergences in microbial roles might result in diversity-and function-changing discrepancies between males and females as colorectal cancer develops; we referred to this scenario as role effects. In addition, colorectal cancer might trigger species replacement in both the male and female gut, and those newly enriched species likely compensated for most ecological functions of depleted species in the male gut, but because of heavy community changes, ecological functions were probably entirely changed; we referred to this scenario as a 'compensatory effect'. In our study, despite species losses occurring in males, no significant difference in a- diversities was observed, and community composition also did not show obvious aberrances with colorectal cancer development proceeding in males (except for a slight divergence between healthy and cancer individuals $\left(\mathrm{R}^{2}=0.063, P\right.$ $<0.05$ ) (Fig. 1A \& 1C)). In addition, specific OTUs and functional genes increased with the development of colorectal cancer in males compared with females. Moreover, contrary to females, higher node counts and stronger connections among network modules also occurred in males. Therefore, we may infer that compensatory effects are more important than role effects in males, whereas role effects play a major role in females with the development of colorectal cancer.

The microbial community assembly pattern revealed how microbes responded to the development of colorectal cancer at the community scale. Previous studies demonstrated that deterministic processes governed microbial community assembly in both healthy and diseased individuals, and host immunity largely determined this process $[38,47,48]$. A recent study indicated that sexual divergence in the immune system largely contributed to microbial colonization in the gut, which in turn further governed the sexual discrepancies in the immune system [12]. From the insights of our current study, the development of colorectal cancer randomized the gut microbial spectrum in male individuals but exerted large filtering pressures on the gut microbial communities in female individuals. We attributed this result to different microbial community assembly mechanisms between male and female individuals. Specifically, although the size of the species pool increased, both $a$-diversity and community composition were not changed or less changed in the male gut with the development of colorectal cancer (Fig. 1A \& C), suggesting that rare species in individuals with colorectal cancer governed the microbial community deviation from healthy individuals. This was because vast rare species in communities would result in a high probability of some ecological events (e.g., ecological drifts associated with random birth and death, replacement of individuals) [49] and further elevated the importance of stochastic processes in the gut of male individuals with colorectal cancer. In contrast, species loss with the development of colorectal cancer in the female gut might deterministically alter the microbial community structure and eventually increase the importance of deterministic processes, which is supported by the fact that the species pool size and $a$ - and $\beta$-diversities decreased with the development of colorectal cancer. These results from our present study implied that the vulnerability of female gut microbial communities to colorectal cancer primarily results from species loss.

Co-occurrence networks inferred from microbial communities in males showed highly complex relationships among microbial taxa compared to those in females, indicating higher microbial 
community stability in the male gut [50]. Indeed, associations among network modules in male networks were increased; however, they were decreased significantly in female networks with the development of colorectal cancer (Fig. 4), as well as the variation of topological properties of gut microbial networks in males and females (Additional file 1: Fig. S4), further supporting our hypothesis. In addition, centralitytier-based analysis showed that rare species enriched in male gut microbial communities might serve as key hubs to maintain microbial community stability during the development of colorectal cancer, and ecological compensatory effects likely improved the inner-or intraspecies interactions in male gut microbial communities to adapt to physiological changes in the host gut [51]. However, depleted species might have important functions and serve as the key nodes in networks in the gut microbial communities of females during the development of colorectal cancer. This supported our hypothesis on role effects. we found that the Parcubacteria phylum (also known as candidate phylum OD1) was located in the intermediate tier of male networks when colorectal adenoma occurred (Fig. 6). According to previous studies, microbes belonging to the Parcubacteria phylum were ectosymbionts or parasites [52]. Although the putative role of these highly adapted organisms in colorectal cancer remains poorly understood [17], it can be suggested that Parcubacteria might largely contribute to the stability of the microbial network at the colorectal adenoma stage in males. In addition, Lentisphaerae, Planctomycetes and Spirochaetes (located in the central and intermediate tiers) were only detected in males with colorectal cancer, showing potential as biomarkers for colorectal cancer in males. Recent studies showed that microbes belonging to the Lentisphaerae or Planctomycetes phyla possibly played a role in carbon recycling and dissolved organic production and acted as an additional source of energy and carbon in the natural environment (such as water) $[53,54]$, inferring that these microbes might possess high adaptability to shifts in the environment and play a vital role in maintaining stability in males with the development of colorectal cancer. Unlike the variation in phyla in the male gut, decreased abundance of the phyla Lentisphaerae and Acidobacteria was observed in the female gut with the development of colorectal cancer, suggesting that these phyla might contribute to the weakening of the network in females.

\section{Conclusions}

We demonstrated that the gut microbial community responds distinctly to the development of colorectal cancer between males and females. Our findings suggest that microbial communities are more stable in the male gut than in the female gut during the development of colorectal cancer, and females likely sustain high risks of shifts in the microbiome, including changes in diversity, community structure, and microbial interactions. Meanwhile, this study highlights an important role of sex for accurate prediction of the risk of colorectal cancer.

\section{Methods}

\section{Data collection}

All 90 fecal samples in the present study were derived from a published project [9]. Raw sequence reads were available at http://www.mothur.org/MicrobiomeBiomarkerCRC. Briefly, fecal samples were randomly 
collected from healthy participants $(n=30)$, colonic adenoma $(n=30)$ and colonic adenocarcinoma patients $(n=30)$ between one and four weeks after colonoscopy preparation. The 16S rRNA genes in each sample were amplified and sequenced using the Illumina MiSeq platform. To compare the differences between male and female individuals, we classified the 90 samples into two groups: male ( $\mathrm{n}$ $=50$, contained healthy, colorectal adenoma and carcinoma individuals $)$ and female $(n=40$, contained healthy, colorectal adenoma and carcinoma individuals).

\section{Bioinformatics analysis}

Raw paired-end reads were assembled and filtered using USEARCH $8.1[55,56]$. Sequences with ambiguous nucleotides, lengths less than 200 bps or expected error values greater than 1 were discarded. Chimeric sequences in our dataset were removed using the UCHIME algorithm, and clean data were clustered into operational taxonomic units (OTUs) at a 3\% cutoff with the UPARSE algorithm. OTU representatives were assigned using the SILVA 16S rRNA gene database [57]. OTUs that were assigned to mitochondrial DNA were removed before further analysis. We resampled 25747 sequences for each sample to ensure the accuracy of the group across comparisons and obtained 8220 OTUs with 9,046,493 sequences in total after removal of singletons. The $a$-diversity indices were calculated using QIIME 1.9.

\section{Ecological statistical analysis}

Gut microbial communities were visualized using principal coordinate analysis (PCoA) with Bray-Curtis dissimilarities, and the overall differences in microbial community composition among developmental stages of colorectal cancer were tested using analysis of similarity (ANOSIM). Microbial $a$-diversities were compared using a random permutation test with 999 iterations. To estimate potential metabolic changes in microbial communities during the development of colorectal cancer, potential functional genes were predicted using the Taxa4Fun R package [58]. Unweighted Venn diagrams were further applied to compare shared and specific OTUs/functional genes among colorectal cancer development stages.

\section{Null model analysis}

Underlying microbial assembly patterns were estimated using the null models [35, 59]. We first calculated microbial community dispersion for the colorectal cancer proceeding stage in males and females based on modified Raup-Crick dissimilarity. This probabilistic dissimilarity metric measured the deviation in community dissimilarities from null distribution under random assembling, which allowed us to distinguish $\beta$-diversity from $\alpha$-diversity [59]. In addition, this metric also implied possible community assembly mechanisms [59]. A high community dispersion implied the high importance of stochastic processes in microbial community assembly; otherwise, deterministic processes might dominate [35]. 
Differences in microbial community dispersion among the developmental stages of colorectal cancer were tested using permutational analysis of multivariate dispersion (PERMDISP, 999 iterations).

The observed and expected Jaccard dissimilarities based on random resampling were further calculated for each developmental stage of colorectal cancer with 999 iterations. The $\beta$-deviation, which was defined as the divergence between observed and mean expected matrices divided by the standard deviation of expected values (standard effect size, SES) and is usually used to measure the real $\beta$-diversity after controlling random sampling effects, was calculated. Differences between observed and expected $\beta$ diversity as well as the $\beta$-deviation divergences among colorectal cancer stages were tested using PERMDISP with 999 iterations. The species pools in both Raup-Crick and Jaccard's dissimilarity-based null models were defined as the total species number in each developmental stage of each gender.

\section{Co-occurrence network analysis}

To evaluate the response of microbial interactions to colorectal cancer, we inferred two meta cooccurrence networks for male and female gut microbial communities. Briefly, the Spearman rank correlation coefficients among OTUs were first calculated, and $P$-values were adjusted using the Benjamini and Hochberg false discovery rate (FDR) [60]. A random matrix theory (RMT) [61]-based approach was used to determine the correlation cutoffs. Co-occurrence networks without selfconnections were inferred using igraph (https://igraph.org/) python package (male: $\rho=0.70 P<0.001$; female: $\rho=0.73 P<0.001$ ). The subnetwork of each sample and development stage of colorectal cancer was split from the meta network by preserving OTUs presented in each sample and group, respectively [62].

Node-level topological properties compassing betweenness and degree centralities were calculated for subnetworks. Nodes in each subnetwork were classified as peripheral, intermediate or central by ranking all nodes according to betweenness centrality, partitioning this ranked list into three equally populated bins, which were termed "centrality tiers" [62]. Network-level topological properties, including node count, edge count, average path length, clustering coefficient, cluster number, modularity, diameter, degree assortativity and density, were further calculated for subnetworks. Topological structures of subnetworks from each developmental stage of colorectal cancer were compared using principal component analysis (PCA), and analysis of similarity (ANOSIM) based on network-level topological indices was calculated. All topological properties were calculated using igraph (https://igraph.org/) python package, and network modules were detected with a greedy modularity optimization algorithm. Differences in subnetwork properties among developmental stages of colorectal cancer were tested using a random permutation test with 999 iterations. Network images were generated using Gephi (http://gephi.github.in/).

\section{Declarations}

\section{Acknowledgments}


We thank Joseph P. Zackular, Joseph P. Zackular, Mary A.M. Rogers, Mack T. Ruffin IV, and Patrick D. Schloss for the contribution of sequence data in current study. They may work in Department of Microbiology and Immunology, University of Michigan, Ann Arbor, Michigan, Department of Internal Medicine, University of Michigan, Ann Arbor, Michigan or Department of Family Medicine, University of Michigan, Ann Arbor, Michigan.

\section{Authors' contributions}

HJL drafted the manuscript and completed data analysis. CNL completed the task of data pre-processing and revised the figures. YWA and YPK critically revised the manuscript. All authors read and approved the final manuscript.

\section{Funding}

This study was supported by the West Light Foundation of Chinese Academy of Sciences (Y8C2041100).

\section{Availability of data and material}

Not applicable

\section{Ethics approval and consent to participate}

Not applicable

\section{Consent for publication}

Not applicable

\section{Competing interests}

The authors declare that they have no competing interests

\section{References}

1. Lasry A, Zinger A, Ben-Neriah Y. Inflammatory networks underlying colorectal cancer. Nat Immunol. 2016;17:230.

2. Tamboli CP, Neut C, Desreumaux P, Colombel JF. Dysbiosis in inflammatory bowel disease. Gut. 2004;53:1-4.

3. Kaiko GE, Stappenbeck TS. Host-microbe interactions shaping the gastrointestinal environment. Trends Immunol. 2014;35:538-548.

4. Dalal SR, Chang EB. The microbial basis of inflammatory bowel diseases. J Clin Invest. 2014;124:4190-4196. 
5. Wang G, Yu Y, Wang YZ, Wang JJ, Guan R, Sun Y, Shi F, Gao J, Fu XL. Role of SCFAs in gut microbiome and glycolysis for colorectal cancer therapy. J Cell Physiol. 2019.

6. Villeger R, Lopes A, Veziant J, Gagniere J, Barnich N, Billard E, Boucher D, Bonnet M. Microbial markers in colorectal cancer detection and/or prognosis. World J Gastroenterol. 2018;24:2327-2347.

7. Sze MA, Schloss PD. Leveraging Existing 16S rRNA Gene Surveys To Identify Reproducible Biomarkers in Individuals with Colorectal Tumors. Mbio. 2018;9:e00630-00618.

8. Zeller G, Tap J, Voigt AY, Sunagawa S, Kultima JR, Costea PI, Amiot A, Bohm J, Brunetti F, Habermann $\mathrm{N}$, et al. Potential of fecal microbiota for early-stage detection of colorectal cancer. Mol Syst Biol. 2014;10:766.

9. Zackular JP, Rogers MA, Ruffin MTt, Schloss PD. The human gut microbiome as a screening tool for colorectal cancer. Cancer Prev Res (Phila). 2014;7:1112-1121.

10. Allen J, Sears CL. Impact of the gut microbiome on the genome and epigenome of colon epithelial cells: contributions to colorectal cancer development. Genome Med. 2019;11:11.

11. Liu S, da Cunha AP, Rezende RM, Cialic R, Wei Z, Bry L, Comstock LE, Gandhi R, Weiner HL. The Host Shapes the Gut Microbiota via Fecal MicroRNA. Cell Host Microbe. 2016;19:32-43.

12. Fransen F, Beek AAV, Borghuis T, Meijer B, Hugenholtz F, Jongh CVDG, Savelkoul HF, Jonge MID, Faas MM, Boekschoten MV. The Impact of Gut Microbiota on Gender-Specific Differences in Immunity. Front Immunol. 2017;8:754.

13. Elin O, Margarete M, Parks BW, Petia S, Liu X, Drake TA, Lusis AJ. Sex differences and hormonal effects on gut microbiota composition in mice. Gut Microbes. 2016;7:313-322.

14. Markle JG, Frank DN, Mortintoth S, Robertson CE, Feazel LM, Rollekampczyk U, Von BM, Mccoy KD, Macpherson AJ, Danska JS. Sex differences in the gut microbiome drive hormone-dependent regulation of autoimmunity. Gut Microbes. 2014;339:1084-1088.

15. Hooper LV, Gordon JI. Commensal host-bacterial relationships in the gut. Science. 2001;292:11151118.

16. Flemer B, Lynch DB, Brown JMR, Jeffery IB, Ryan FJ, Claesson MJ, O'Riordain M, Shanahan F, O'Toole PW. Tumour-associated and non-tumour-associated microbiota in colorectal cancer. Gut. 2017;66:633-643.

17. Thomas AM, Jesus EC, Lopes A, Samuel Aguiar J, Begnami MD, Rocha RM, Carpinetti PA, Camargo AA, Hoffmann C, Freitas HC. Tissue-Associated Bacterial Alterations in Rectal Carcinoma Patients Revealed by $16 S$ rRNA Community Profiling. Frontiers in Cellular \& Infection Microbiology. 2016;6:179.

18. Uemura N, Okamoto S, Yamamoto S, Matsumura N, Yamaguchi S, Yamakido M, Taniyama K, Sasaki N, Schlemper RJ. Helicobacter pylori Infection and the Development of Gastric Cancer. New Engl J Med. 2001;345:784-789.

19. Ahn J, Sinha R, Pei Z, Dominianni C, Wu J, Shi J, Goedert JJ, Hayes RB, Yang L. Human Gut Microbiome and Risk for Colorectal Cancer. Jnci-Journal of the National Cancer Institute. 2013;105:1907-1911. 
20. Zhao D, Liu H, Zheng Y, He Y, Lu D, Lyu C. A reliable method for colorectal cancer prediction based on feature selection and support vector machine. Med Biol Eng Comput. 2019;57:901-912.

21. Feng Q, Liang S, Jia H, Stadlmayr A, Tang L, Lan Z, Zhang D, Xia H, Xu X, Jie Z, et al. Gut microbiome development along the colorectal adenoma-carcinoma sequence. Nat Commun. 2015;6:6528.

22. Mira-Pascual L, Cabrera-Rubio R, Ocon S, Costales P, Parra A, Suarez A, Moris F, Rodrigo L, Mira A, Collado MC. Microbial mucosal colonic shifts associated with the development of colorectal cancer reveal the presence of different bacterial and archaeal biomarkers. J Gastroenterol. 2015;50:167-179.

23. Odamaki T, Kato K, Sugahara H, Hashikura N, Takahashi S, Xiao JZ, Abe F, Osawa R. Age-related changes in gut microbiota composition from newborn to centenarian: a cross-sectional study. BMC Microbiol. 2016;16:90.

24. Koistinen VM, Karkkainen O, Borewicz K, Zarei I, Jokkala J, Micard V, Rosa-Sibakov N, Auriola S, Aura AM, Smidt $H$, Hanhineva K. Contribution of gut microbiota to metabolism of dietary glycine betaine in mice and in vitro colonic fermentation. Microbiome. 2019;7:103.

25. Carmody RN, Gerber GK, Jr JML, Gatti DM, Somes L, Svenson KL, Turnbaugh PJ. Diet dominates host genotype in shaping the murine gut microbiota. Cell Host \& Microbe. 2015;17:72-84.

26. Kovacs A, Ben-Jacob N, Tayem H, Halperin E, Iraqi FA, Gophna U. Genotype Is a Stronger Determinant than Sex of the Mouse Gut Microbiota. Microb Ecol. 2011;61:423-428.

27. van Nas A, Guhathakurta D, Wang SS, Yehya N, Horvath S, Zhang B, Ingram-Drake L, Chaudhuri G, Schadt EE, Drake TA, et al. Elucidating the role of gonadal hormones in sexually dimorphic gene coexpression networks. Endocrinology. 2009;150:1235-1249.

28. Takagi T, Naito Y, Inoue R, Kashiwagi S, Uchiyama K, Mizushima K, Tsuchiya S, Dohi O, Yoshida N, Kamada K, et al. Differences in gut microbiota associated with age, sex, and stool consistency in healthy Japanese subjects. J Gastroenterol. 2019;54:53-63.

29. Wang J, Wang J, Pang X, Zhao L, Tian L, Wang X. Sex differences in colonization of gut microbiota from a man with short-term vegetarian and inulin-supplemented diet in germ-free mice. Sci Rep. 2016;6:36137.

30. Min Y, Ma X, Sankaran K, Ru Y, Chen L, Baiocchi M, Zhu S. Sex-specific association between gut microbiome and fat distribution. Nat Commun. 2019;10:2408.

31. Yurkovetskiy L, Burrows M, Khan AA, Graham L, Volchkov P, Becker L, Antonopoulos D, Umesaki Y, Chervonsky AV. Gender Bias in Autoimmunity Is Influenced by Microbiota. Immunity. 2013;39:400412.

32. Markle JG, Frank DN, Mortin-Toth S, Robertson CE, Feazel LM, Rolle-Kampczyk U, von Bergen M, McCoy KD, Macpherson AJ, Danska JS. Sex differences in the gut microbiome drive hormonedependent regulation of autoimmunity. Science. 2013;339:1084-1088.

33. Chen JJ, Zheng P, Liu YY, Zhong XG, Wang HY, Guo YJ, Xie P. Sex differences in gut microbiota in patients with major depressive disorder. Neuropsychiatr Dis Treat. 2018;14:647-655.

34. Tripathi BM, Stegen JC, Kim M, Dong K, Adams JM, Lee YK. Soil pH mediates the balance between stochastic and deterministic assembly of bacteria. Isme j. 2018;12:1072-1083. 
35. Zhou J, Deng Y, Zhang P, Xue K, Liang Y, Van Nostrand JD, Yang Y, He Z, Wu L, Stahl DA, et al. Stochasticity, succession, and environmental perturbations in a fluidic ecosystem. Proceedings of the National Academy of Sciences of the United States of America. 2014;111:E836-E845.

36. Wang J, Shen J, Wu Y, Tu C, Soininen J, Stegen JC, He J, Liu X, Zhang L, Zhang E. Phylogenetic beta diversity in bacterial assemblages across ecosystems: deterministic versus stochastic processes. Isme Journal. 2013;7:1310-1321.

37. Kraft NJ, Comita LS, Chase JM, Sanders NJ, Swenson NG, Crist TO, Stegen JC, Vellend M, Boyle B, Anderson $\mathrm{MJ}$, et al. Disentangling the drivers of beta diversity along latitudinal and elevational gradients. Science. 2011;333:1755-1758.

38. Dai L, Kou H, Xia Y, Wen X, Gao J, Ma ZS. Does colorectal cancer significantly influence the assembly of gut microbial communities? PeerJ. 2017;5:e3383.

39. Kang M, Martin A. Microbiome and colorectal cancer: Unraveling host-microbiota interactions in colitis-associated colorectal cancer development. Semin Immunol. 2017;32:3-13.

40. Drago L, Toscano M, De GR, Grossi E, Padovani EM, Peroni DG. Microbiota network and mathematic microbe mutualism in colostrum and mature milk collected in two different geographic areas: Italy versus Burundi. Isme Journal. 2017;11:875-884.

41. Kara EL, Hanson PC, Hu YH, Winslow L, McMahon KD. A decade of seasonal dynamics and cooccurrences within freshwater bacterioplankton communities from eutrophic Lake Mendota, WI, USA. Isme Journal. 2013;7:680-684.

42. Faust K, Sathirapongsasuti JF, Izard J, Segata N, Gevers D, Raes J, Huttenhower C. Microbial cooccurrence relationships in the human microbiome. PLoS Comput Biol. 2012;8:e1002606.

43. Steele JA, Countway PD, Xia L, Vigil PD, Beman JM, Kim DY, Chow C-ET, Sachdeva R, Jones AC, Schwalbach MS, et al. Marine bacterial, archaeal and protistan association networks reveal ecological linkages. Isme Journal. 2011;5:1414-1425.

44. Ibrahim A, Hugerth LW, Hases L, Saxena A, Seifert M, Thomas Q, Gustafsson JA, Engstrand L, Williams C. Colitis-induced colorectal cancer and intestinal epithelial estrogen receptor beta impact gut microbiota diversity. Int J Cancer. 2019;144:3086-3098.

45. Huang R, Li T, Ni J, Bai X, Gao Y, Li Y, Zhang P, Gong Y. Different Sex-Based Responses of Gut Microbiota During the Development of Hepatocellular Carcinoma in Liver-Specific Tsc1-Knockout Mice. Front Microbiol. 2018;9:1008.

46. Logares R, Mangot J-F, Massana R. Rarity in aquatic microbes: placing protists on the map. Res Microbiol. 2015;166:831-841.

47. Sun Y, Li L, Xia Y, Li W, Wang K, Wang L, Miao Y, Ma S. The gut microbiota heterogeneity and assembly changes associated with the IBD. Sci Rep. 2019;9.

48. Jeraldo P, Sipos M, Chia N, Brulc JM, Dhillon AS, Konkel ME, Larson CL, Nelson KE, Qu A, Schook LB, et al. Quantification of the relative roles of niche and neutral processes in structuring gastrointestinal microbiomes. Proceedings of the National Academy of Sciences of the United States of America. 2012;109:9692-9698. 
49. Burns AR, Stephens WZ, Stagaman K, Wong S, Rawls JF, Guillemin K, Bohannan BJM. Contribution of neutral processes to the assembly of gut microbial communities in the zebrafish over host development. Isme Journal. 2016;10:655-664.

50. Fan K, Weisenhorn P, Gilbert JA, Chu H. Wheat rhizosphere harbors a less complex and more stable microbial co-occurrence pattern than bulk soil. Soil Biol Biochem. 2018;125:251-260.

51. Greenblum S, Turnbaugh PJ, Borenstein E. Metagenomic systems biology of the human gut microbiome reveals topological shifts associated with obesity and inflammatory bowel disease. Proc Natl Acad Sci U S A. 2012;109:594-599.

52. Nelson WC, Stegen JC. The reduced genomes of Parcubacteria (OD1) contain signatures of a symbiotic lifestyle. Front Microbiol. 2015;6:713.

53. Spring S, Bunk B, Sproer C, Schumann P, Rohde M, Tindall BJ, Klenk HP. Characterization of the first cultured representative of Verrucomicrobia subdivision 5 indicates the proposal of a novel phylum. Isme j. 2016;10:2801-2816.

54. Yilmaz P, Yarza P, Rapp JZ, Glockner FO. Expanding the World of Marine Bacterial and Archaeal Clades. Front Microbiol. 2015;6:1524.

55. Edgar RC, Haas BJ, Clemente JC, Quince C, Knight R. UCHIME improves sensitivity and speed of chimera detection. Bioinformatics. 2011;27:2194-2200.

56. Edgar RC. Search and clustering orders of magnitude faster than BLAST. Bioinformatics. 2010;26:2460-2461.

57. Pruesse E, Quast C, Knittel K, Fuchs BM, Ludwig W, Peplies J, Gloeckner FO. SILVA: a comprehensive online resource for quality checked and aligned ribosomal RNA sequence data compatible with ARB. Nucleic Acids Res. 2007;35:7188-7196.

58. Asshauer KP, Wemheuer B, Daniel R, Meinicke P. Tax4Fun: predicting functional profiles from metagenomic 16S rRNA data. Bioinformatics. 2015;31:2882-2884.

59. Chase JM, Kraft NJB, Smith KG, Vellend M, Inouye BD. Using null models to disentangle variation in community dissimilarity from variation in alpha-diversity. Ecosphere. 2011;2.

60. Benjamini Y, Krieger AM, Yekutieli D. Adaptive linear step-up procedures that control the false discovery rate. Biometrika. 2006;93:491-507.

61. Luo F, Zhong J, Yang Y, Scheuermann RH, Zhou J. Application of random matrix theory to biological networks. Physics Letters A. 2006;357:420-423.

62. Ma B, Wang H, Dsouza M, Lou J, He Y, Dai Z, Brookes PC, Xu J, Gilbert JA. Geographic patterns of cooccurrence network topological features for soil microbiota at continental scale in eastern China. Isme j. 2016;10:1891-1901.

\section{Additional File Legends}

Tab. S1 The topological features of male and female meta-community co-occurrence networks. 
Fig. S1 The appearance/disappearance of all observed OTUs with the development of colorectal carcinoma in the gut microbial communities of males.

Fig. S2 The appearance/disappearance of all observed OTUs with the development of colorectal carcinoma in the gut microbial communities of females.

Fig. S3 Principal component analysis of network-level topological features in the male (A) and female (B) gut microbial co-occurrence networks.

Fig. S4 The network-level topological features in the microbial subnetwork of the male and female gut with the development of colorectal carcinoma.

\section{Figures}
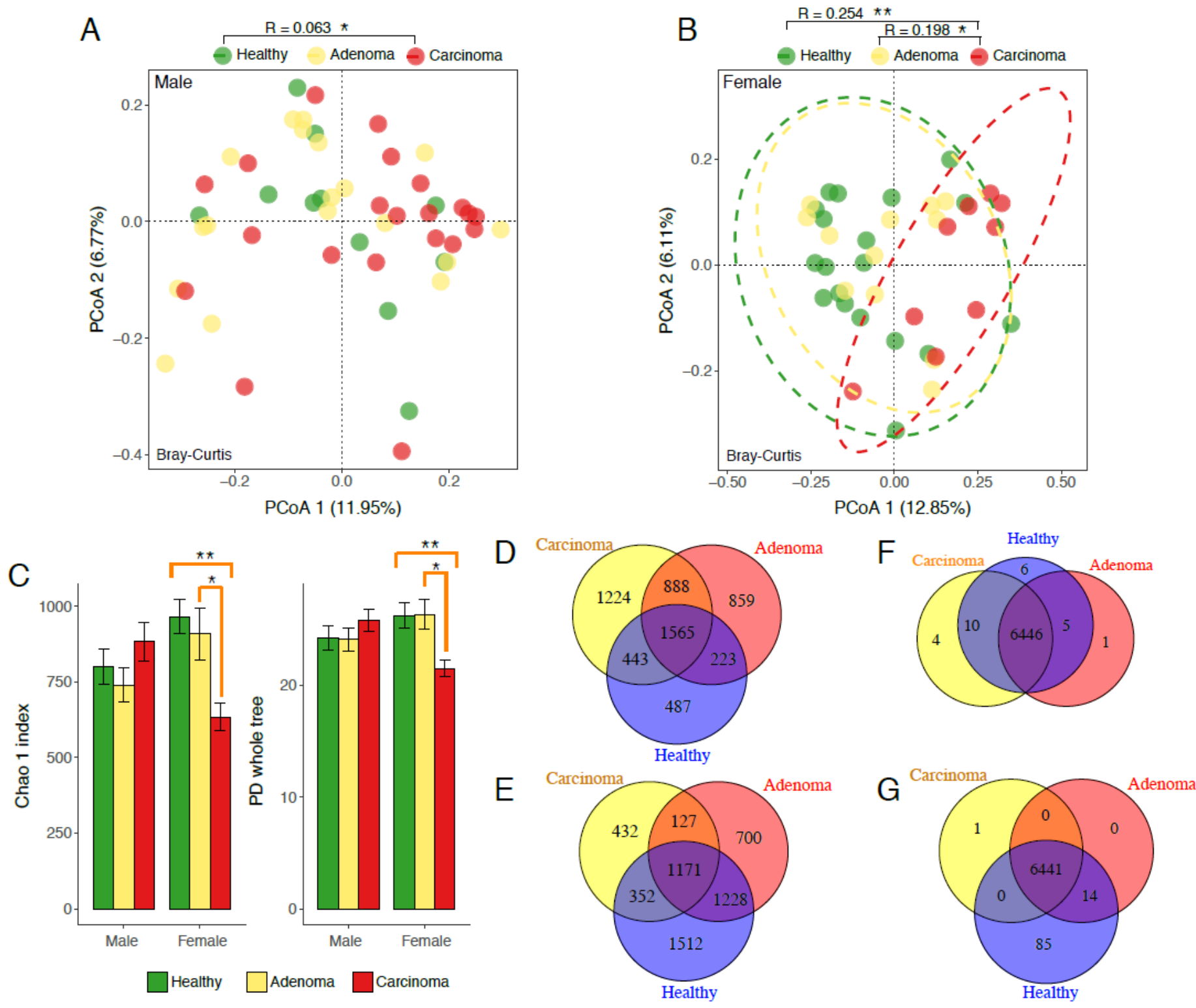

Figure 1 
The gut microbial community structure of male (A) and female (B) individuals based on Bray-Curtis dissimilarities; the changes in the gut microbial a-diversity in response to the development of colorectal carcinoma in both genders (C); the unweighted OTU spectrum that was overlapped and differentiated in the male (D) and female (E) gut, and the unweighted microbial enzyme spectrum overlapped and differentiated in the male (F) and female (G) gut. The differences in (A) and (B) were tested using analysis of similarity (ANOSIM), while in (C), the significance test method was the random permutation test (RPT). ${ }^{\star} \mathrm{P}<0.05, * \star P<0.01, * \star \star P<0.001$.
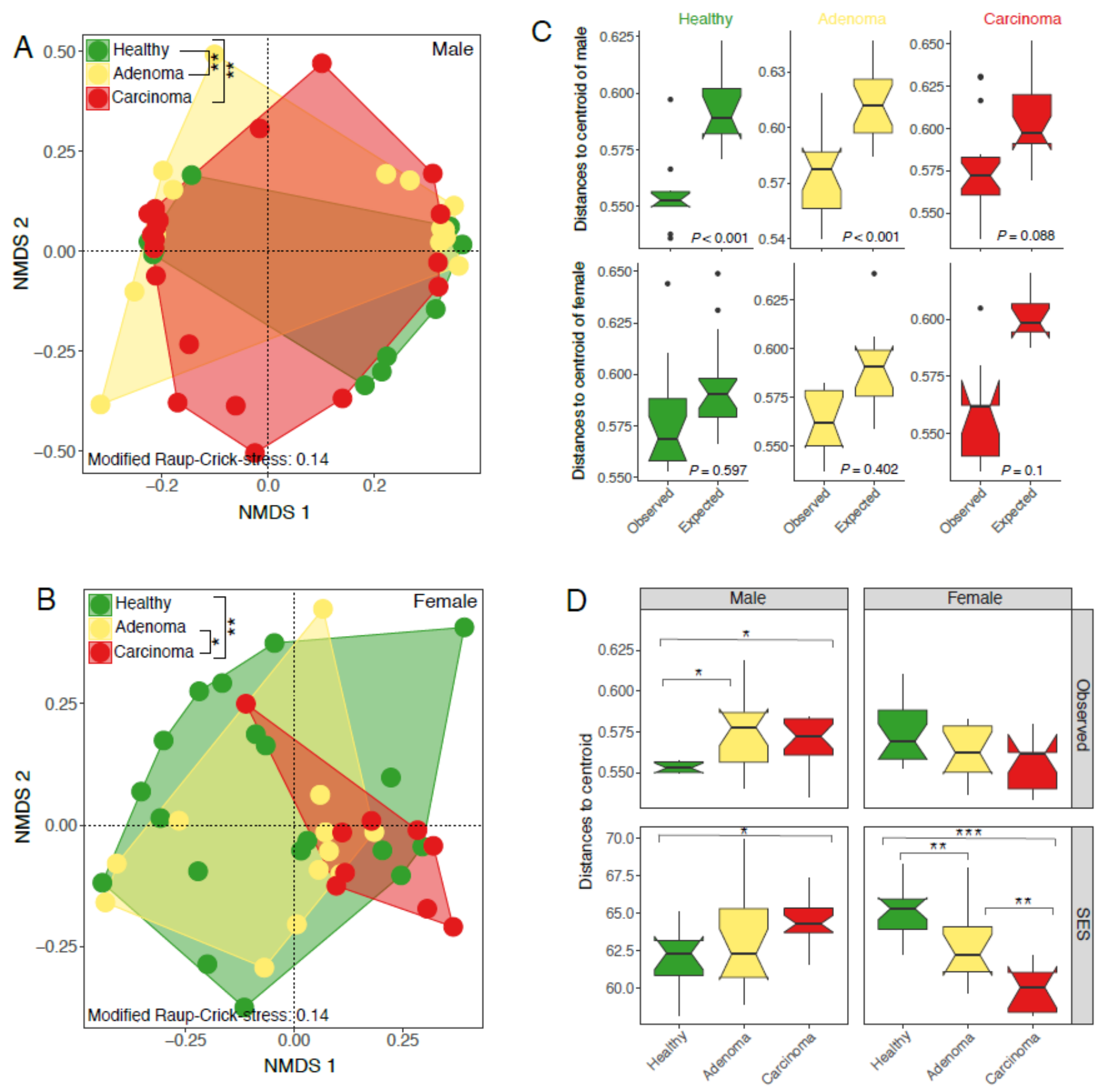

Figure 2 
Nonmetric multidimensional scaling (NMDS) based on modified Raup-Crick dissimilarities for male (A) and female (B) gut microbial communities; the assembly pattern variation in gut microbial communities in male and female (C) individuals; the variation of the observed $\beta$-diversity and $\beta$-deviation with changes in the clinical status (D). The differences of community dispersion among groups were tested using permutational analysis of multivariate dispersion with 999 iterations. ${ }^{*} \mathrm{P}<0.05,{ }^{*} \mathrm{P}<0.01$, $* * * \mathrm{P}<0.001$.

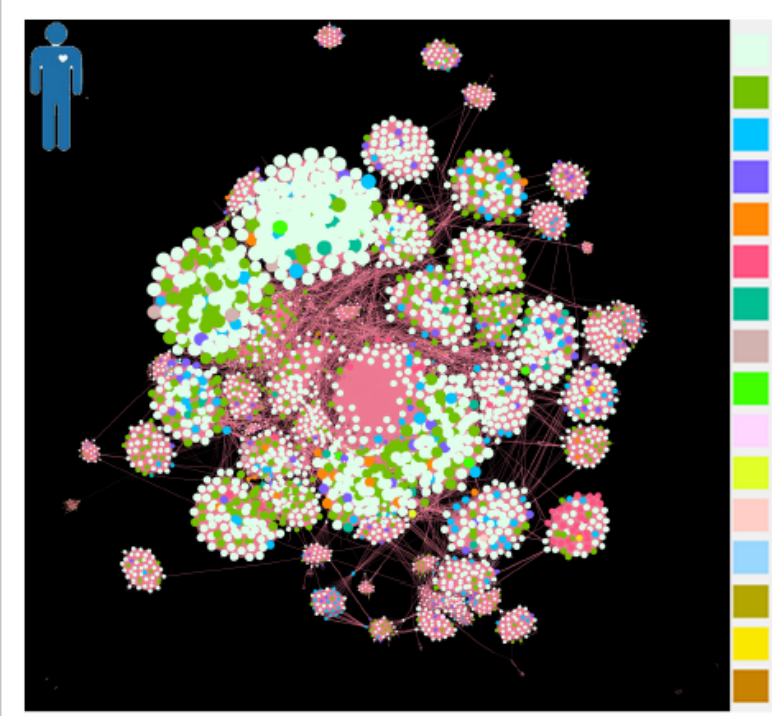

$\begin{array}{ll}\text { p: Firmicutes } & (69.78 \%) \\ \text { p: Bacteroidetes } & (18.37 \%) \\ \text { p: Proteobacteria } & (4.45 \%) \\ \text { p:Actinobacteria } & (3.96 \%) \\ \text { p: Verrucomicrobia } & (0.79 \%) \\ \text { p: Fusobacteria } & (0.77 \%) \\ \text { p: Tenericutes } & (0.7 \%) \\ \text { p: Cyanobacteria } & (0.4 \%) \\ \text { p: Euryarchaeota } & (0.28 \%) \\ \text { p: Deinococcus-Thermus } & (0.21 \%) \\ \text { p:Synergistetes } & (0.14 \%) \\ \text { p:Saccharibacteria } & (0.07 \%) \\ \text { p: Planctomycetes } & (0.02 \%) \\ \text { p: Parcubacteria } & (0.02 \%) \\ \text { p: Spirochaetae } & (0.02 \%) \\ \text { p: Lentisphaerae } & (0.02 \%)\end{array}$
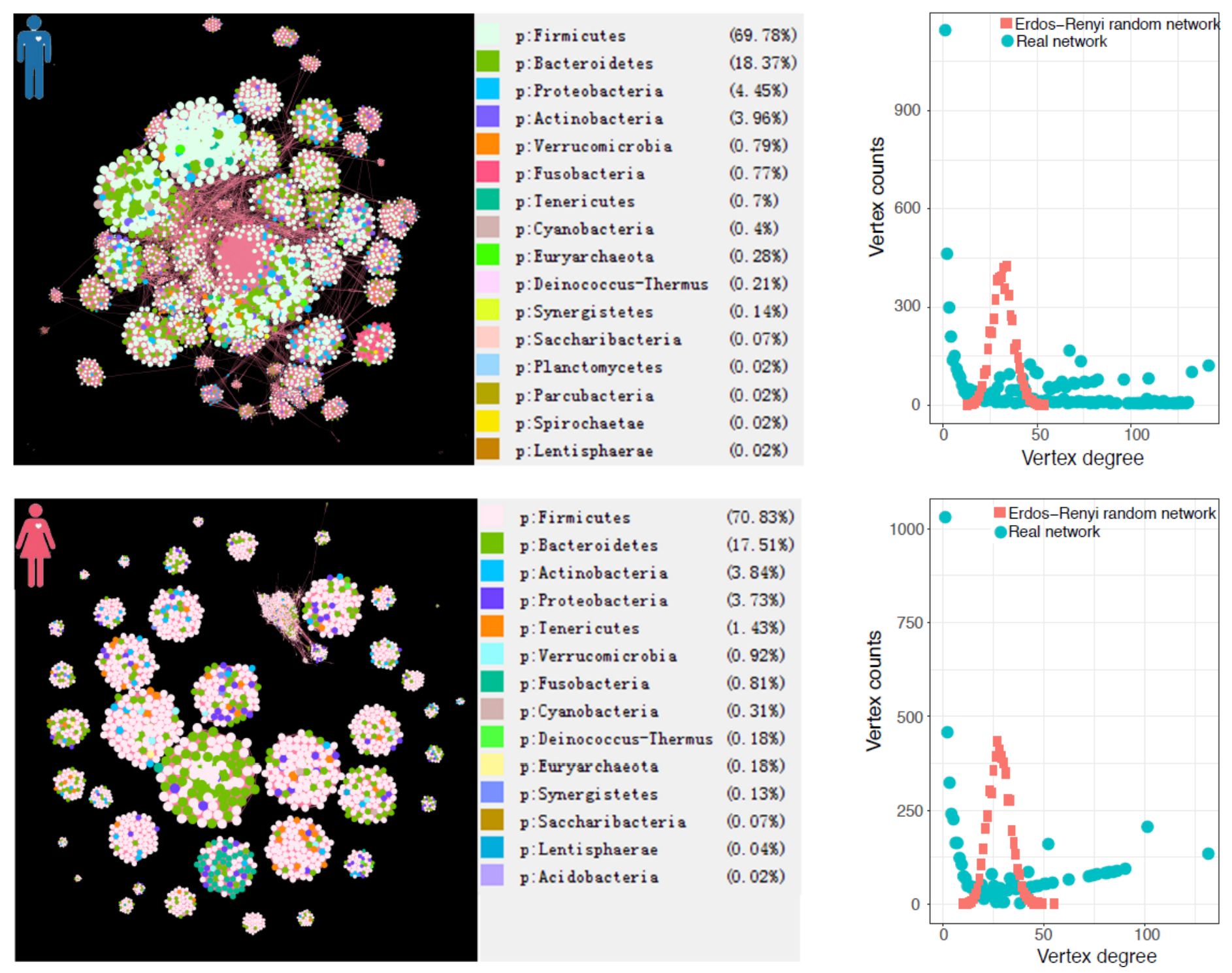

Figure 3

The microbial meta-community co-occurrence networks and associated degree distribution in the male ( $\rho$ $=0.70, P<0.001)$ and female $(\rho=0.73, P<0.001)$ gut. The nodes in the network represent OTUs in the community, and the edges represent the correlations among OTUs. All nodes were colored based on microbial phyla. The isolated nodes or nodes with extremely low degree centrality may not be seen in these two networks. 
Healthy
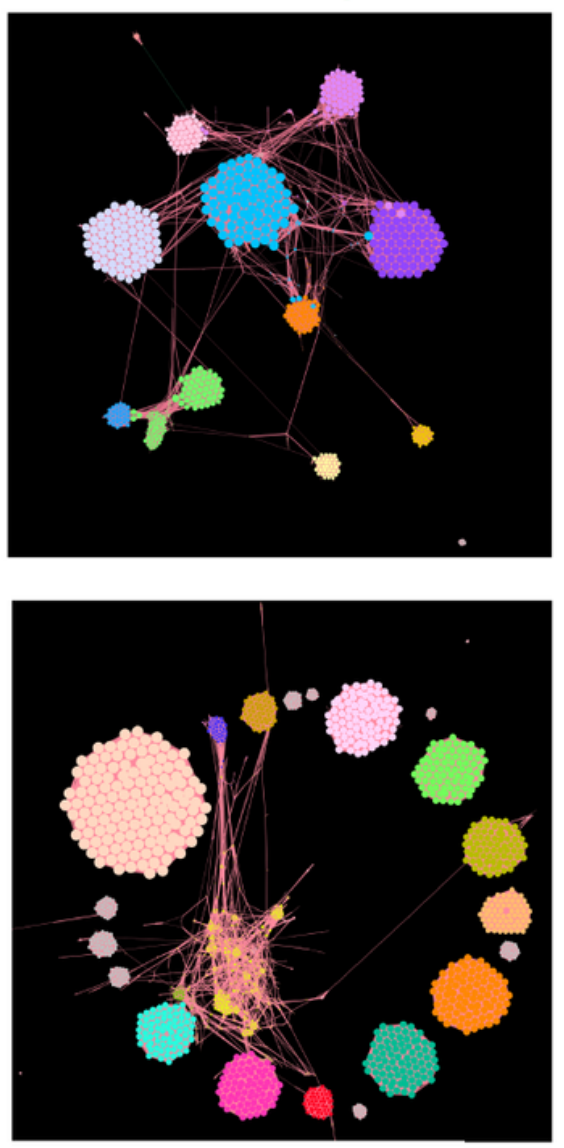

Adenoma
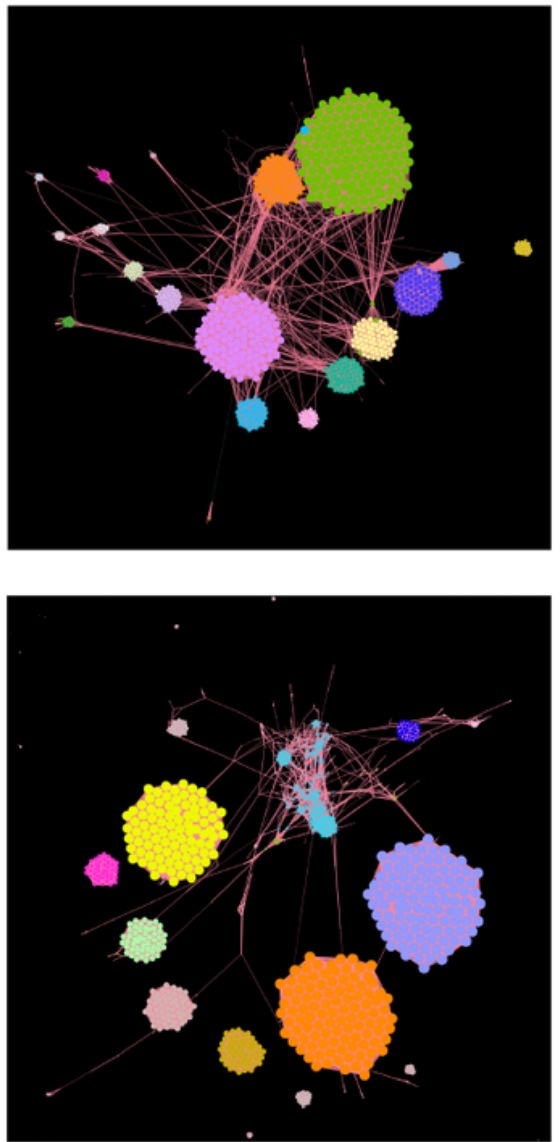

Carcinoma
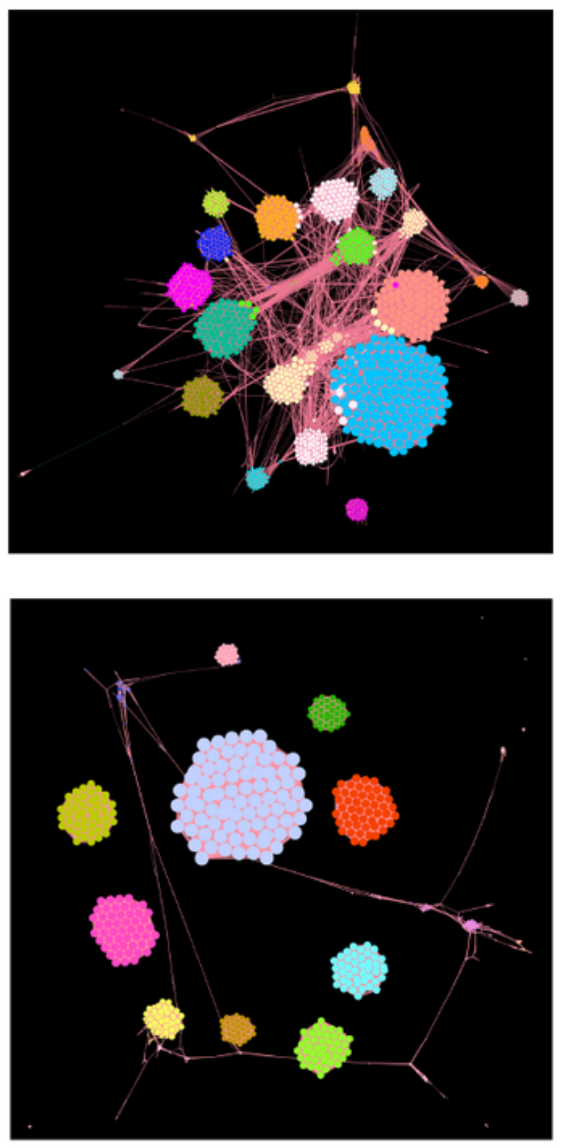

\section{Figure 4}

The microbial cooccurrence network variations with the development of colorectal carcinoma in the male and female gut. The nodes in the network represent OTUs in the community, and the edges represent the correlations among OTUs. All nodes were colored based on network modules (only modules with nodes with an abundance greater than $1 \%$ in the network are colored); those isolated nodes or nodes with extremely low degree centrality may not be seen in these two networks. 

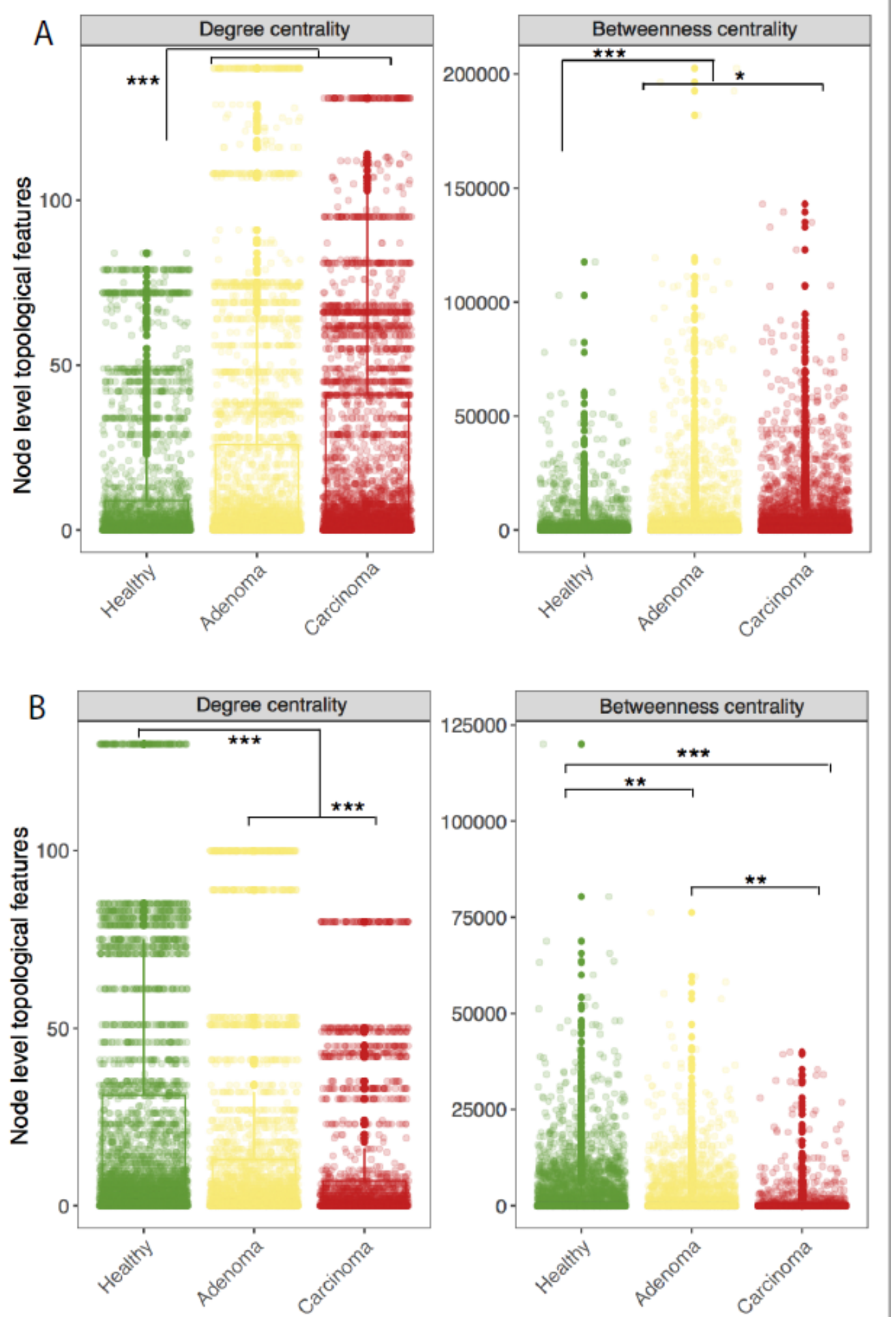

\section{Figure 5}

Changes in the degree and betweenness centrality of microbial cooccurrence networks during the development of colorectal carcinoma in the male (A) and female gut (B). The significance tests were carried out using a random permutation test (RPT). ${ }^{\star} P<0.05$, ${ }^{\star \star} P<0.01$, ${ }^{\star \star \star} P<0.001$. 


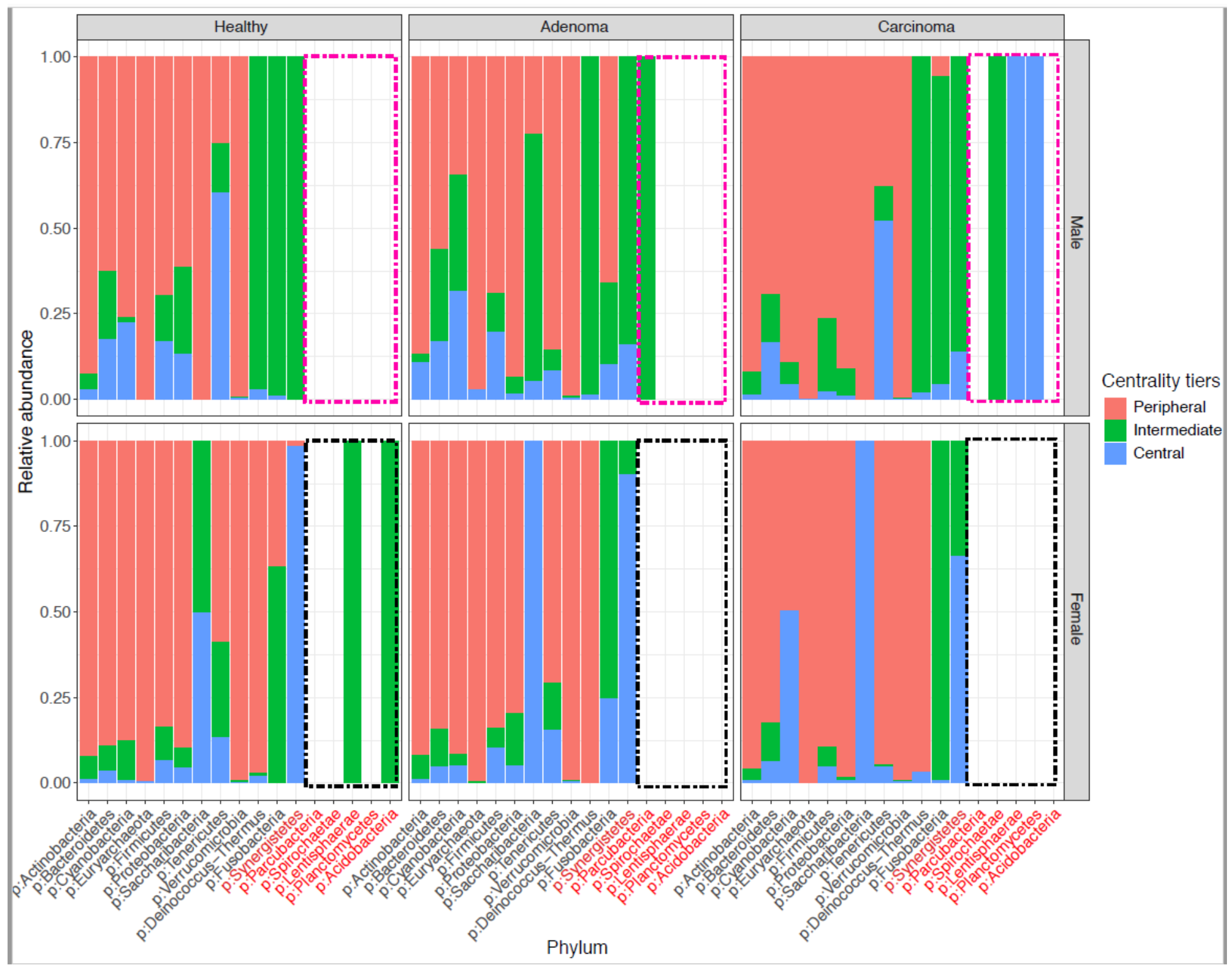

\section{Figure 6}

The percentage of nodes belonging to different phyla in different centrality tiers. These relative positions of each node in the network were inferred using betweenness centrality. The nodes with high betweenness centrality are closer to the center of the network compared to those with low betweenness centrality, and vice versa.

\section{Supplementary Files}

This is a list of supplementary files associated with this preprint. Click to download.

- Additionalfile1.docx 